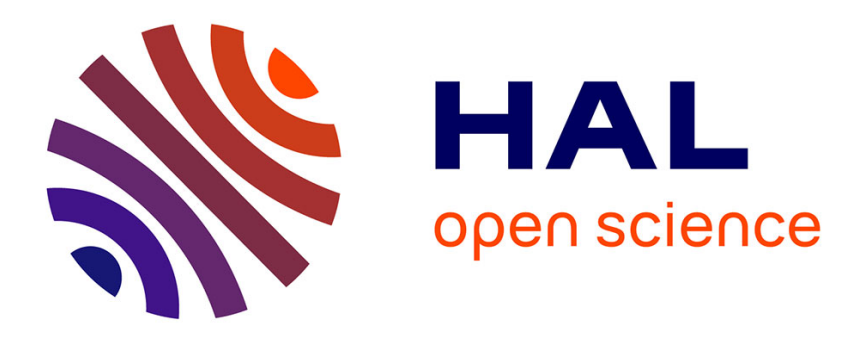

\title{
Polarimétrie acoustique ultrasonore. Application à l'acousto-élasticimétrie
}

\author{
A. Zarembowitch, E. Khalifa
}

\section{To cite this version:}

A. Zarembowitch, E. Khalifa. Polarimétrie acoustique ultrasonore. Application à l'acousto-élasticimétrie. Revue de Physique Appliquée, 1985, 20 (6), pp.359-363. 10.1051/rphysap:01985002006035900 . jpa-00245344

\section{HAL Id: jpa-00245344 https://hal.science/jpa-00245344}

Submitted on 1 Jan 1985

HAL is a multi-disciplinary open access archive for the deposit and dissemination of scientific research documents, whether they are published or not. The documents may come from teaching and research institutions in France or abroad, or from public or private research centers.
L'archive ouverte pluridisciplinaire HAL, est destinée au dépôt et à la diffusion de documents scientifiques de niveau recherche, publiés ou non, émanant des établissements d'enseignement et de recherche français ou étrangers, des laboratoires publics ou privés. 


\title{
Polarimétrie acoustique ultrasonore. Application à l'acousto-élasticimétrie
}

\author{
A. Zarembowitch $(*)$ et E. Khalifa $\left({ }^{*}\right)\left({ }^{* *}\right)$ \\ (*) Laboratoire de Recherches Physiques (Laboratoire associé au CNRS no 71), Tour 22, Université Pierre et \\ Marie Curie, 4, place Jussieu, 75230 Paris Cedex 05, France \\ (**) Société T.E.I., 7, rue du Chant des Oiseaux, 78360 Montesson, France
}

(Reçu le 29 novembre 1984, accepté le 26 décembre 1984)

\begin{abstract}
Résumé. - En se fondant sur les analogies qui existent entre les ondes élastiques transversales ultrasonores se propageant dans les solides et les ondes électromagnétiques, on peut développer l'équivalent acoustique de la photoélasticimétrie.

On décrit dans l'article un acousto-élasticimètre qui permet, sans changer l'orientation des transducteurs émetteurs et récepteurs, de déterminer la biréfringence acoustique et l'orientation des axes principaux de biréfringence par mesure de fréquence.

Abstract. - By using the analogy between ultrasonic shear elastic waves propagating in solids and electromagnetic waves, it is possible to develop the acoustical analogue of photoelasticimetry.

In this paper we describe an acousto elasticimeter which allows, by frequency measurements, the determination of the acoustical birefringence and the orientation of the principal birefringence axis, without changing the orientation of the transmitting and receiving transducers.
\end{abstract}

\section{Introduction.}

La propagation des ondes élastiques dans les milieux fluides ou solides présente beaucoup d'analogies avec celle des ondes électromagnétiques. En particulier, cette parenté est étroite pour ce qui concerne les propriétés de polarisation des ondes lumineuses et celles des ondes transversales se propageant dans les solides : tous les phénomènes de l'optique électromagnétique classique ont un équivalent acoustique : effet Faraday, polarisation rotatoire, effet Pockels, réfraction conique... [1-5].

On peut fonder sur ces ressemblances formelles une polarimétrie acoustique inspirée des phénomènes et des techniques utilisées en polarimétrie optique.

En fait, l'intérêt d'une telle approche n'a pas échappé aux chercheurs en physique appliquée, mais la mise en œuvre pratique de telles méthodes se heurte à des difficultés technologiques importantes : pour transmettre des ondes élastiques transversales dans un solide, on peut, soit utiliser sous incidence oblique lá conversion de modes longitudinaux en modes trans- versaux, mais alors les vibrations transversales sont polarisées uniquement dans le plan d'incidence, soit employer, sous incidence normale, un «liant " visqueux qui autorise la rotation du polariseur. Dans ce cas, la durée de la relaxation du liant visqueux interdit pratiquement le contrôle continu de pièces mécaniques que l'on ferait défiler devant le transducteur émetteur. Ces obstacles ont freiné pendant près de 20 ans le développement d'une polarimétrie ultrasonore dont les principes fondamentaux étaient cependant largement connus.

Aujourd'hui, l'importance économique croissante des problèmes de contrôle de matériaux rend utile toute méthode d'analyse et d'évaluation des contraintraintes, fût-elle une évaluation locale dans laquelle les sondes ultrasonores sont fixées à demeure en des points déterminés de la structure dont on veut contrôler l'évolution de l'état de contrainte.

Nous avons donc étudié la possibilité de réaliser un polarimètre ultrasonore (acoustoélasticimètre) à sondes fixes. Ce travail prolonge une étude réalisée dans les laboratoires de la Société Thomson [6]. 


\section{Biréfringence acoustique.}

Considérons un solide isotrope de forme parallélépipédique soumis à une contrainte uniaxiale uniforme qui s'exerce sur les faces perpendiculaires à $\mathrm{O} y$ (Fig. 1).

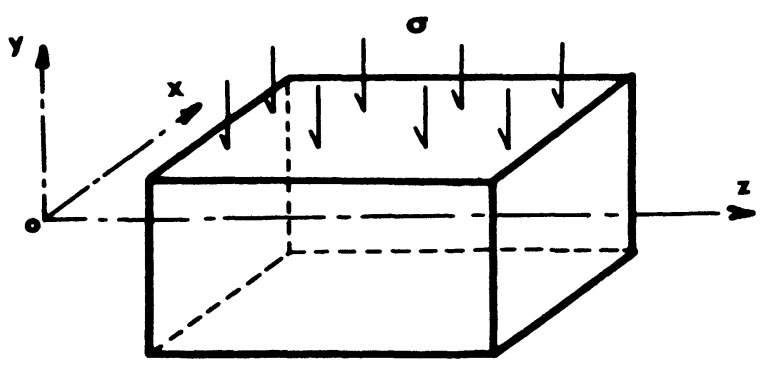

Fig. 1. - Parallélépipède rectangle soumis à une contrainte uniaxiale.

[Parallelepiped with a uniaxial stress.]

Les vitesses des ondes élastiques transversales se propageant suivant $\mathrm{O} z$ sont différentes selon que la polarisation est parallèle à $\mathrm{O} y$ ou à $\mathrm{O} x$.

On a, pour l'onde polarisée parallèlement à $\mathrm{O} y$ :

$V_{\mathrm{t}_{1}}=V_{0}\left(1-\frac{\sigma}{\mu(3 \lambda+2 \mu)}\left(2 \mu+\lambda+m+\frac{n \lambda}{4 \mu}\right)\right)^{1 / 2}$

pour l'onde polarisée parallèlement à $O x$ :

$V_{\mathrm{t}_{2}}=V_{0}\left(1-\frac{\sigma}{\mu(3 \lambda+2 \mu)}\left(m-2 \lambda-\frac{\lambda+\mu}{2 \mu} n\right)\right)^{1 / 2}$

où

$\lambda$ et $\mu$ sont les constantes élastiques du $2^{\mathrm{e}}$ ordre de Lame,

$m, n$ sont les constantes élastiques du $3^{\mathrm{e}}$ ordre,

$V_{0}$ la vitesse des ondes élastiques transversales du milieu non contraint,

$\sigma$ la contrainte uniaxiale.

Par analogie avec l'optique électromagnétique, on désigne ce phénomène sous le nom "d'effet Pockels acoustique " ou de "biréfringence acoustique provoquée ». Notons que dans des milieux naturellement anisotropes on peut avoir, pour certaines directions de propagation, des modes de vibration purement transversaux et par conséquent "une biréfringence acoustique naturelle». Un milieu primitivement isotrope peut aussi conserver une «biréfringence rémanente " quand il a été soumis à de fortes contraintes (laminage à froid).

$\mathrm{Au}$ plan expérimental, on peut prolonger cette analogie avec l'optique, car on sait réaliser, dans le domaine acoustique ultrasonore des transducteurs piézoélectriques engendrant et détectant des vibrations transversales; ces transducteurs. sont les équivalents des polariseurs et analyseurs optiques.
Une vibration élastique transversale excitée à l'aide d'un transducteur polariseur et se propageant suivant $\mathrm{O} z$ est en général transformée à la sortie du solide en une vibration elliptique. Analyser cette vibration elliptique qui caractérise l'état de contrainte (supposé uniforme) du solide, c'est faire de l'acoustoélasticimétrie analogue acoustique de la photoélasticimétrie. Notons que le déphasage $\varphi$ introduit entre vibration " lente » et "rapide " par la traversée du milieu contraint s'exprime simplement à l'aide du seul coefficient du $3^{\mathrm{e}}$ ordre $n$ dans le cas où $\varphi$ est petit devant $2 \pi$

$$
\varphi=\frac{\omega e \sigma}{2 V_{0} \mu}\left(1+\frac{n}{4 \mu}\right)
$$

avec : $e$ épaisseur traversée, $\omega$ pulsation de l'onde.

Dans la pratique on rencontrera souvent des situations expérimentales plus complexes, modélisables cependant par une suite de milieux biréfringents que l'onde élastique transversale doit traverser successivement. Le traitement théorique de ce problème est facilité par l'emploi de la sphère de Poincaré dont nous rappelons brièvement quelques propriétés.

Soit une vibration elliptique repérée par rapport à des axes $\mathrm{O} x, \mathrm{O} y$ (Fig. 2). Son équation paramétrique est :

$$
\begin{aligned}
& x=\cos \rho \cdot \cos \omega t \\
& y=\sin \rho \cdot \cos (\omega t-\psi) .
\end{aligned}
$$

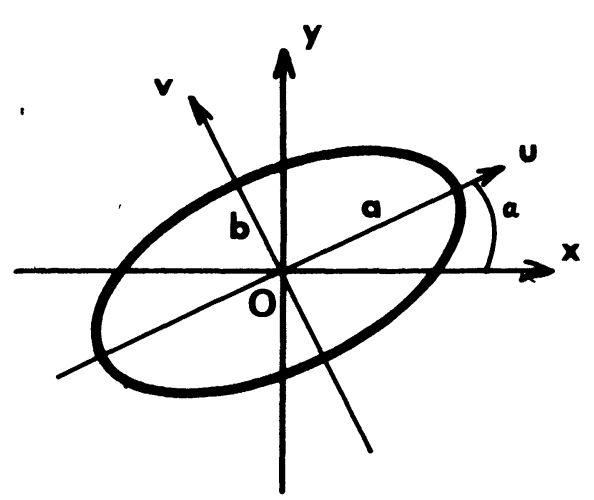

Fig. 2. - Orientation des axes principaux de biréfringence.

[Orientation of the principal axis of birefringence.]

On désignera par :

$\alpha$ l'angle des axes $\mathrm{O} x, \mathrm{O} u$,

$a, b$ les longueurs des demi-axes,

$\lambda$ l'angle tel que $\operatorname{tg} \lambda=b / a$; le signe de $\lambda$ définit le sens de parcours sur l'ellipse.

Un point $M$ de la sphère de Poincaré (Fig. 3) repéré $\operatorname{par}(2 \alpha, 2 \lambda)$ ou par $(\psi$ et $2 \rho)$ caractérise la vibration elliptique.

On sait que l'hémisphère supérieur (inférieur) représente les vibrations droites (gauches). Les pôles repré- 


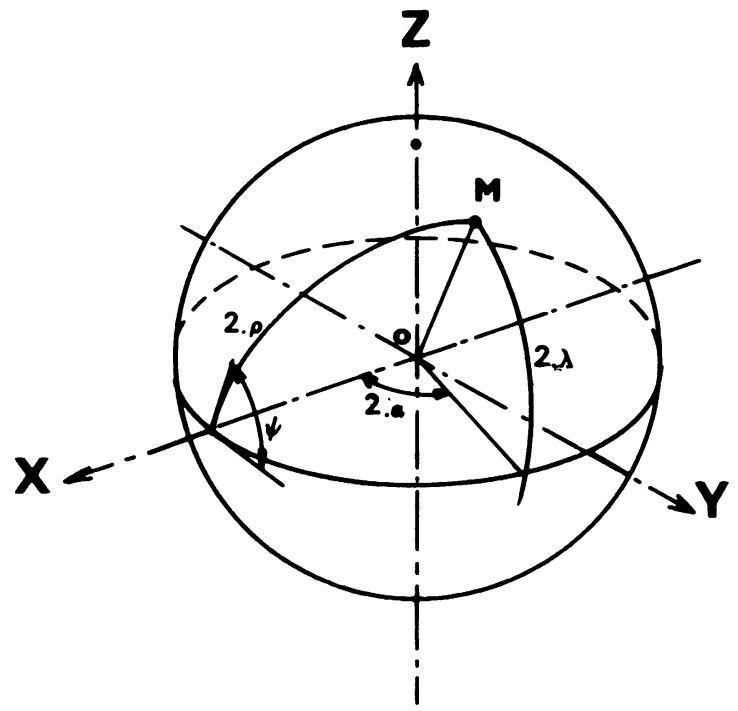

Fig. 3. - Caractéristiques d'un milieu biréfringent représentées sur la sphère de Poincaré.

[Characteristics of a birefringent medium shown on a Poincaré sphere.]

sentent des vibrations circulaires. L'équateur est associé aux vibrations rectilignes.

La traversée d'une suite de milieux biréfringents se traduit analytiquement par la composition de rotations d'axe $\mathrm{O} z$ (pouvoir rotatoire) et d'axes équatoriaux.

A l'aide de la sphère de Poincaré, le théorème suivant peut facilement être démontré :

l'effet d'une suite de lames biréfringentes quelconques est équivalent à celui d'une lame biréfringente unique suivie d'une rotation d'axe $\mathrm{Z}$.

\section{Principe d'un acousto-élasticimètre.}

Suivant la nature de la situation à analyser, plusieurs solutions de complexités croissantes peuvent être retenues ; cependant, pour toutes les configurations examinées, le milieu contraint sera supposé avoir la forme d'un parallélépipède uniformément contraint (Fig. 1).

$1^{\mathrm{er}}$ cas : on connaît les directions des axes principaux de contrainte du milieu étudié.

Soient $\mathrm{O} x$ et $\mathrm{O} y$ ces directions. La seule inconnue est la biréfringence $B=\frac{V_{t_{1}}-V_{t_{2}}}{V_{0}}$, ou le déphasage $\varphi=\frac{\omega e B}{V_{0}}$.

On réalise la chaîne acoustique suivante (Fig. 4).

Soient $\mathbf{P}$ et $\mathbf{A}$ les transducteurs émetteurs et récepteurs; si $\mathrm{A}$ est orthogonal à $\mathrm{P}$, le signal reçu sur $\mathrm{A}$ s'annule pour toutes les fréquences $v$ telles que : $\varphi=2 p \pi, p$ entier, ou

$$
v=p \frac{V_{0}}{e \sigma} \frac{8 \mu^{2}}{4 \mu+n} .
$$

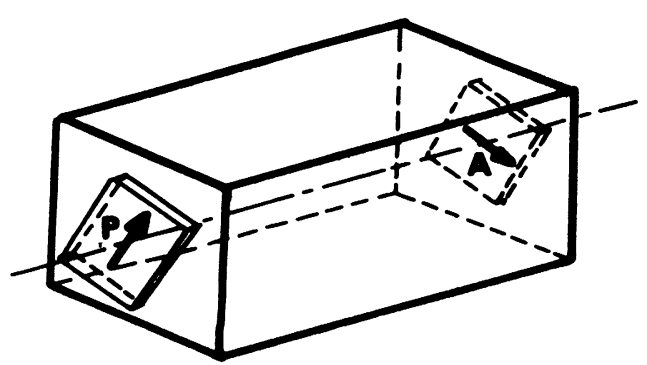

Fig. 4. - Transducteurs ultrasonores analogues à des polariseurs croisés.

[Ultrasonic transducers equivalent to crossed polarizer.]

Dans le cas d'une lame naturellement biréfringente (ex. : lame de fluorine) taillée perpendiculairement à une direction $|110|$ du cristal et pour une épaisseur de la lame $e=2,16 \mathrm{~mm}$, la suite des fréquences $v$ (A perpendiculaire à $\mathrm{P}$ ) forme une progression arithmétique de raison $v=7,3 \mathrm{MHz}$.

Remarque : si la biréfringence est faible, le principe de la mesure reste valable, mais l'intervalle $\Delta v$ peut être de plusieurs mégahertz; dans ce cas certains points expérimentaux se situent dans un domaine de fréquence où l'atténuation ultrasonore devient prohibitive. On peut sans difficulté se ramener au cas précédent en faisant suivre (ou précéder) le milieu biréfringent inconnu par un milieu connu de biréfringence suffisamment forte pour que $\Delta v<1 \mathrm{MHz}$.

$2^{\mathrm{e}}$ cas : la biréfringence et les directions des axes principaux de biréfringence du milieu étudié sont inconnues.

Soient $O x^{\prime}$ et $O y^{\prime}$ ces directions, $\varphi$ le déphasage introduit par le milieu. Si, comme précédemment, la biréfringence inconnue est faible, il est utile de lui additionner une biréfringence connue assez forte pour situer les mesures dans le domaine de fréquence conve-

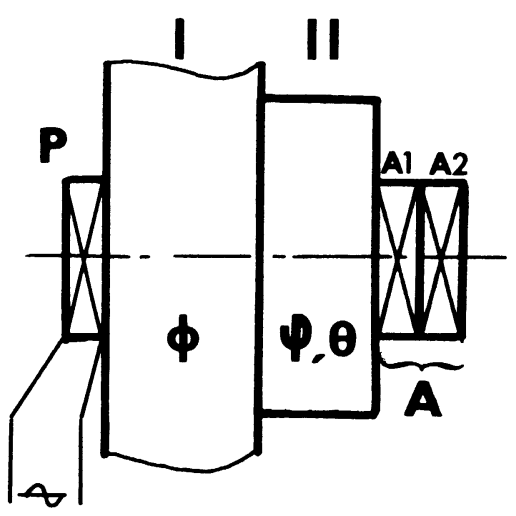

Fig. 5. - Chaîne acoustique comprenant : un polariseur, deux milieux biréfringents I et II, un polariseur composite formé de deux transducteurs croisés $A_{1}$ et $A_{2}$.

[Acoustic chain with : a polarizer, two birefringent media I and II, a composite polarizer $A$ made with two crossed transducers $A_{1}$ and $A_{2}$.] 


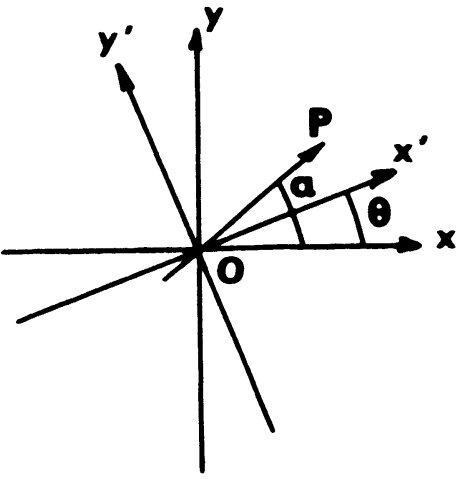

Fig. 6. - Orientation du polariseur $\mathrm{P}$ par rapport aux milieux biréfringents I et II.

[Orientation of the polarizer $\mathbf{P}$ with respect to birefringent media I and II.] nable. Nous traiterons donc directement le cas général constitué par la chaîne acoustique représentée figures 5 et 6 .

Soient $\mathrm{O} x$ et $\mathrm{O} y$ les directions des axes rapides et lents du milieu biréfringent connu, introduisant un déphasage $\phi$.

L'effet des deux lames biréfringentes I et II est équivalent aux effets successifs d'une lame biréfringente unique introduisant un déphasage $\psi$ et d'une rotation d'angle $R . \mathrm{O} u, \mathrm{O} v$ désignant les directions des axes rapide et lent de la lame biréfringente unique équivalente, on notera :

$$
\begin{aligned}
& \gamma=\widehat{\mathrm{Ox, \textrm {O } u}} \\
& \theta=\widehat{\mathrm{Ox, \textrm {O } x}} .
\end{aligned}
$$

En utilisant la sphère de Poincaré, on démontre aisément les relations suivantes :

$$
\begin{gathered}
\operatorname{tg} 2 \gamma=\frac{\sin \varphi \cdot \sin 2 \theta}{\sin \varphi \cdot \cos 2 \theta \cdot \cos \phi+\cos \varphi \cdot \sin \phi} \\
\operatorname{tg} \psi=\frac{\left[\sin ^{2} \varphi \cdot \sin ^{2} 2 \theta+(\sin \varphi \cdot \cos 2 \theta \cdot \cos \phi+\cos \varphi \cdot \sin \phi)^{2}\right]^{1 / 2}}{(\cos \varphi \cdot \cos \phi-\sin \varphi \cdot \cos 2 \theta \cdot \sin \phi)} \\
R=\operatorname{Arg}\left(\operatorname{tg} \frac{\varphi}{2} \cdot \operatorname{tg} \frac{\phi}{2} \cdot \mathrm{e}^{2 i \theta}-1\right) .
\end{gathered}
$$

Cherchons maintenant les conditions pour lesquelles une vibration rectiligne émise par le transducteur $P$ suivant une direction faisant un angle $\alpha$ avec $O x$ demeure rectiligne à la sortie de la chaîne $\mathrm{P}+\mathrm{I}+\mathrm{II}$.

Trois types de solutions peuvent être trouvées :

a) $\phi=2 k \pi, k$ entier.

Cette solution triviale correspond soit à une configuration où $\alpha=0$, soit, si $\alpha \neq 0$ à des fréquences d'excitation du transducteur pour lesquelles la lame I est onde.

L'ensemble I + II se réduit alors à une lame biréfringente unique avec $\psi=\varphi ; R=0 ; \gamma=\theta$.

Cette lame transforme une vibration rectiligne en une vibration rectiligne pour toutes les fréquences pour lesquelles elle est onde ou demi-onde.

b) $\left\{\begin{array}{l}\phi=k \pi, k \text { entier } \\ \varphi=k \pi, k \text { entier }\end{array}\right.$

à cette solution est associée une configuration originale pour les fréquences telles que la lame I et la lame II sont demi-ondes. La lame équivalente est onde et l'effet résultant sur une vibration rectiligne se réduit à une rotation $R=2 \theta$.

c) Il existe une suite de fréquences pour lesquelles la direction $\mathrm{O} u$ de la lame biréfringente coïncide avec la direction $\alpha$ du polariseur $P$.

La vibration émergeant de la chaîne acoustique est alors rectiligne.
Cette configuration correspond à la relation $\operatorname{tg} 2 \gamma=\operatorname{tg} 2 \alpha$ ou

$$
\frac{\sin 2 \theta}{\operatorname{tg} 2 \alpha \cos \phi}=\cos 2 \theta+\frac{\operatorname{tg} \phi}{\operatorname{tg} \varphi} .
$$

Un cas particulier remarquable est celui où $\alpha=n \frac{\pi}{4}$, $n$ entier; alors

$$
\cos 2 \theta+\frac{\operatorname{tg} \phi}{\operatorname{tg} \varphi}=0 .
$$

\section{Résultats.}

La polarimétrie ultrasonore peut recevoir de nombreuses applications, en particulier dans le domaine de la mesure des contraintes résiduelles ou appliquées, sous réserve toutefois que les états de contrainte soient bidimensionnels. Pour illustrer les possibilités d'application, il convient de fixer quelques ordres de grandeur :

- Contraintes appliquées : La formule (3) permet d'exprimer le déphasage introduit entre les deux vibrations $\mathrm{O} x$ et $\mathrm{O} y$ de pulsation $\omega$ (Fig. 1) par la traversée d'une lame d'épaisseur $e$ soumise à une contrainte uniaxiale. Posons $\varphi=a \sigma$. Le tableau I donne des valeurs de $a$ pour une lame d'épaisseur $e=1 \mathrm{~cm}$ et pour divers matériaux (d'après [7]). 
Tableau I

\begin{tabular}{|c|c|c|c|c|}
\hline & $\mu(\mathrm{GPa})$ & $n(\mathrm{GPa})$ & $V_{0} \mathrm{~m} \mathrm{~s}^{-1}$ & $a\left(\operatorname{deg} . \mathrm{Hb}^{-1}\right)$ \\
\hline $\mathrm{Al}$ & 28,3 & $-\quad 280$ & 3237 & $-0,579$ \\
\hline $\mathrm{Cu}$ & 75,3 & -1600 & 2908 & $-0,709$ \\
\hline $\mathrm{Fe}$ & 117 & -2800 & 3850 & $-0,177$ \\
\hline $\mathrm{Ni}$ & 122 & $-\quad 168$ & 3723 & $+0,520$ \\
\hline W & 157 & -480 & 2855 & $+0,019$ \\
\hline
\end{tabular}

- Déformation rémanente : Un milieu primitivement isotrope peut conserver une biréfringence rémanente quand il a été soumis à de fortes contraintes (laminage à froid). Ainsi avons-nous mesuré à $2 \mathrm{MHz}$ le déphasage introduit par la traversée d'une lame d'acier A 36 laminé à $33 \%$ de son épaisseur.

Pour une épaisseur de $1 \mathrm{~cm}$ on trouve :

$V_{\mathrm{t}_{1}}=3250 \mathrm{~m} \cdot \mathrm{s}^{-1}$ dans la direction du laminage

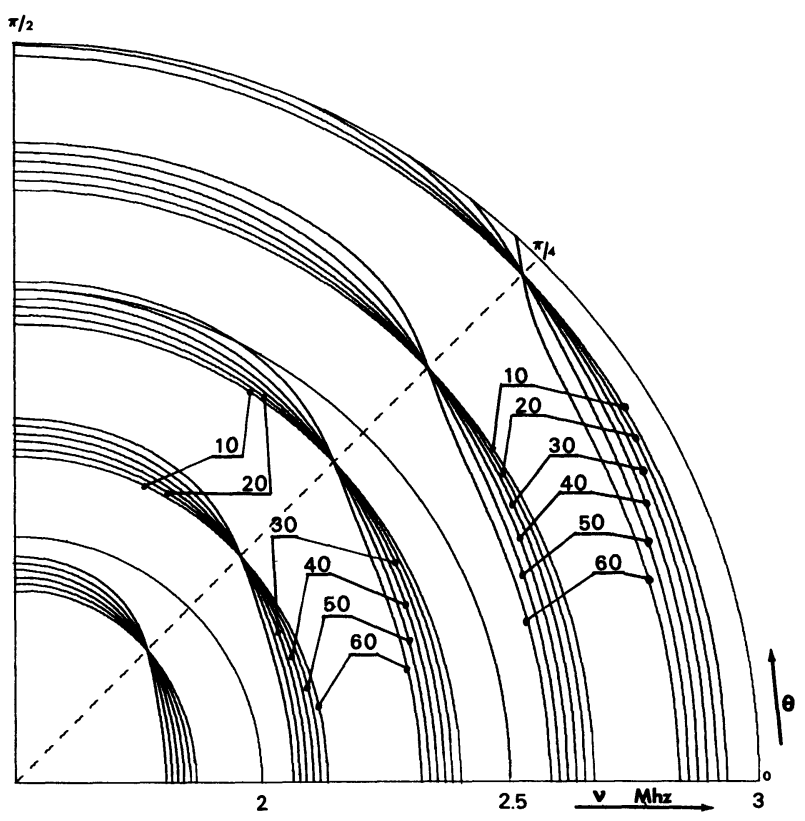

Fig. 8. - Détermination graphique de $\varphi$ et $\theta ; \phi=1350^{\circ}$. [Graphic determination of $\varphi$ and $\theta ; \phi=1350^{\circ}$.]

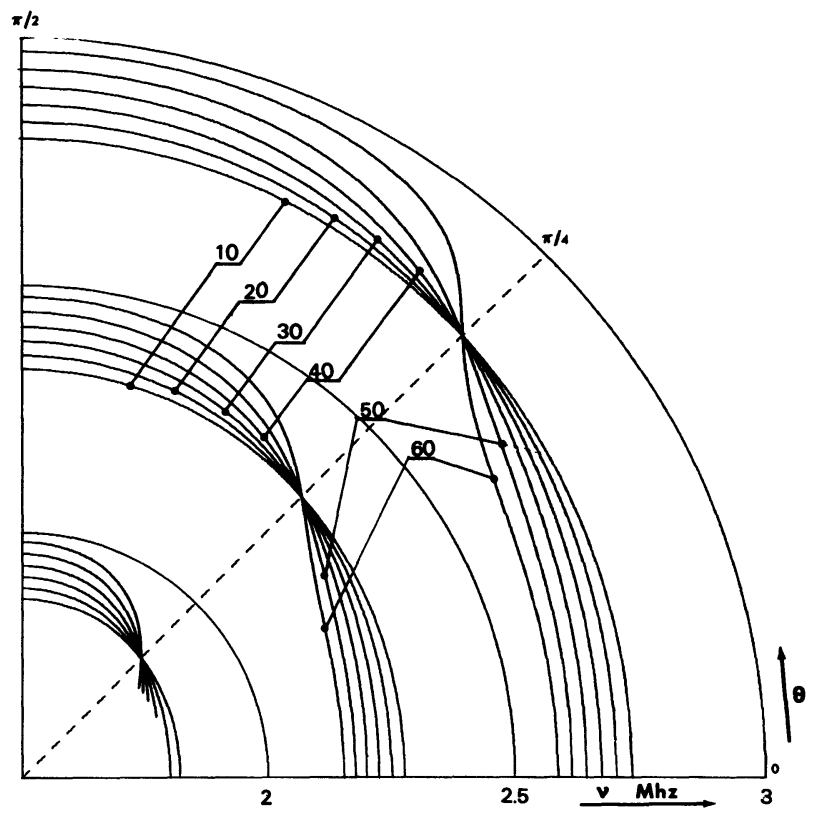

Fig. 7. - Détermination graphique de $\varphi$ et $\theta ; \phi=780^{\circ}$. [Graphic determination of $\varphi$ and $\theta ; \phi=780^{\circ}$.]

$V_{\mathrm{t}_{2}}=3150 \mathrm{~m} \cdot \mathrm{s}^{-1}$ dans la direction perpendiculaire au laminage, soit $\varphi=70^{\circ}$.

La recherche des fréquences successives $v$ pour lesquelles la lame est onde ou demi-onde donne une progression arithmétique de raison $\Delta v=5,10 \mathrm{MHz}$. Dans la pratique, on rencontrera des déphasages $\varphi$ s'échelonnant de quelques degrés à quelques dizaines, voire quelques centaines de degrés.

- Détermination graphique de $\varphi$ et $\theta$ : Afin de pouvoir déterminer $\varphi$ et $\theta$ à partir de la suite des fréquences solution de l'équation (8), il est commode d'utiliser une méthode graphique. Sur les figures 7,8 on a tracé sur un rayon vecteur d'angle polaire $\theta$ les valeurs de $v$ solutions de l'équation (8), pour différentes valeurs de $\varphi: 10,20,30 \ldots 60^{\circ}$.

La figure 7 se rapporte au cas où $\phi=780^{\circ}$.

La figure 8 se rapporte au cas où $\phi=1350^{\circ}$.

\section{Bibliographie}

[1] Pauthier, S., Ann. Phys. Fr. 14 (1966) 195.

[2] FisCher, M. et ZAREMBOWITCH, A., Acustica 285 (1973) 259.

[3] GREEN, R. E., Treatise on materials science and Techno$\log y$, vol. 3 (Acad. Press) 1973.

[4] LJamov, V. E., Effets de polarisation et anisotropie de l'interaction des ondes acoustiques dans les cristaux (Ed. Université de Moscou) 1983.
[5] ZAREMBOWITCH, A., Image processing and coherence in physics (Springer-Verlag) 1980, p. 112.

[6] SociÉTÉ THOMSON CSF, Brevets 80/01895-82/05966.

[7] (LANDOLT, BÖRNSTEIN), vol. II. 